Cite this: Soft Matter, 2014, 10, 3667

\title{
Induced phagocytic particle uptake into a giant unilamellar vesicle
}

\begin{abstract}
Andreas Meinel, ${ }^{a}$ Benjamin Tränkle, ${ }^{a}$ Winfried Römer ${ }^{\text {bc }}$ and Alexander Rohrbach ${ }^{\star a}$
Phagocytosis, the uptake and ingestion of solid particles into living cells, is a central mechanism of our immune system. Due to the complexity of the uptake mechanism, the different forces involved in this process are only partly understood. Therefore the usage of a giant unilamellar vesicle (GUV) as the simplest biomimetic model for a cell allows one to investigate the influence of the lipid membrane on the energetics of the uptake process. Here, a photonic force microscope (PFM) is used to approach an optically trapped $1 \mu \mathrm{m}$ latex bead to an immobilized GUV to finally insert the particle into the GUV. By analysing the mean displacement and the position fluctuations of the trapped particle during the uptake process in 3D with nanometre precision, we are able to record force and energy profiles, as well as changes in the viscous drag and the stiffness. After observing a global followed by a local deformation of the GUV, we measured uptake energies of $2000 \mathrm{kT}$ to $5500 \mathrm{kT}$ and uptake forces of $4 \mathrm{pN}$ to $16 \mathrm{pN}$ for Egg-PC GUVs with sizes of $18-26 \mu \mathrm{m}$ and varying membrane tension. The measured energy profiles, which are compared to a Helfrich energy model for local and global deformation, show good coincidence with the theoretical results. Our proof-of-principle study opens the door to a large number of similar experiments with GUVs containing more biochemical components and complexity. This bottom-up strategy should allow for a better understanding of the physics of phagocytosis.
\end{abstract}

Received 26th November 2013

Accepted 17th February 2014

DOI: $10.1039 /$ c3sm52964a

www.rsc.org/softmatter simplest biomimetic variant is a giant unilamellar vesicle (GUV), ${ }^{10}$ where the chemical and mechanical properties of the spherical lipid bilayer membrane can be controlled in various manners. ${ }^{\mathbf{1 1 - 1 6}}$ In order to engulf and internalize a particle, the spherical membrane of the GUV needs to be deformed significantly, as measured and modelled by several groups. ${ }^{\mathbf{8 1 7 - 2 2}}$

Among various experimental techniques for GUV manipulation, ${ }^{\mathbf{1 0}}$ optical tweezers are especially advantageous due to their ability to apply 3D forces between femto-Newtons ${ }^{23}$ and nano-Newtons ${ }^{24}$ without mechanical contact. In this way Dietrich et $a l .{ }^{17}$ demonstrated that it is possible to ingest 7-18 $\mu \mathrm{m}$ large spheres into GUVs by using double beam optical tweezers. Later, effects of particle adhesion, diffusion, and coupling with GUVs have been studied with single beam optical tweezers. ${ }^{10}$ In combination with a fast 3D tracking system such as back-focal plane (BFP) interferometry the thermal position fluctuations of the trapped particle ${ }^{25,26}$ can be tracked nanometre precise at several $100 \mathrm{kHz}$ - nearby living cells or in contact with them. ${ }^{27}$ Using BFP-interferometry, also the position fluctuations of lipid membranes have been measured in a broad dynamic range to estimate membrane properties. ${ }^{28}$

However, the measurement of force and energy profiles during the induced phagocytic uptake has not been achieved so far, which would enable a comparison to existing theories.

In the present study we describe the controlled internalization of a $1 \mu \mathrm{m}$ small spherical particle into a GUV. Using 3D optical tweezers and 3D BFP-tracking, we were able to measure
${ }^{a}$ Department of Microsystems Engineering (IMTEK), University of Freiburg, 79110 Freiburg, Germany. E-mail: rohrbach@imtek.de

${ }^{b}$ Institute of Biology II, Albert-Ludwigs-University Freiburg, 79104 Freiburg, Germany ${ }^{c}$ BIOSS - Centre for Biological Signalling Studies, Albert-Ludwigs-University Freiburg, 79104 Freiburg, Germany 
distance resolved forces and energies required for particle uptake. In parallel, we recorded the fluctuations of the particle on a $\mu$ s time scale prior to binding, but also during uptake (in contact with the membrane) allowing us in both cases to extract the changes in viscosity and elasticity of the particle-GUV system. Our method allows a systematic comparison of energy profiles between GUV systems with different lipid compositions, but also comparisons to theoretical approaches.

\section{Theoretical background and methods}

\subsection{Vesicle growth by electroformation}

GUVs were prepared from egg phosphatidylcholine (Egg-PC) (Sigma-Aldrich), utilizing the method of electroformation. ${ }^{29}$ Therefore the lipids were dissolved in chloroform resulting in a stock concentration of $0.5 \mathrm{mg} \mathrm{ml}^{-1}$. In order to perform fluorescence microscopy, $1 \mathrm{~mol} \%$ fluorescent lipid analogues (Bodipy FL-C5-HPC, Molecular Probes) were added to the stock solution. For electroformation, an ITO glass chamber was prepared using two facing ITO-coverslips that were coated with an $8 \mu$ ldroplet of the stock solution. ${ }^{\mathbf{1 4 , 3 0}}$ The vesicle formation was accomplished in a 300 mOsm sucrose solution by applying an alternating electric field ( $3 \mathrm{~V} \mathrm{RMS}, 10 \mathrm{~Hz}$ ) for a period of $2 \mathrm{~h}$. The frequency was subsequently reduced to $4 \mathrm{~Hz}$ for $1 \mathrm{~h}$ enhancing the detachment of the formed vesicles from the ITO glass chamber.

\subsection{Reduction of membrane tension}

In order to enable complete particle uptake, the membrane tension of the produced GUVs had to be reduced by decreasing their internal pressure. An intermediate evaporation of the GUV solution droplet during preparation induces an osmotic pressure difference, which subsequently vanishes through the flux of solution molecules from the interior of the vesicles to the extracellular space. A method to measure the membrane tension is GUV fluctuation spectroscopy, ${ }^{28}$ where thermal membrane fluctuations are analysed by their power spectral densities (PSD).

\subsection{Experimental setup}

The phagocytic particle uptake enabled by the optical trap is sketched in Fig. 1a. A $1 \mu \mathrm{m}$ polystyrene particle (Polysciences Inc.) is trapped by a focused laser beam $\left(\lambda_{0}=1064 \mathrm{~nm}, \mathrm{NA}=\right.$ 1.2, water) at powers between 10 and $50 \mathrm{~mW}$ inside the chamber. The $3 \mathrm{D}$ position tracking of the particle relative to the trap center is achieved by BFP-interferometry at rates of 0.1-1 MHz using a quadrant photodiode (QPD). ${ }^{27}$ The motion of the GUV is hindered by $20 \mu \mathrm{m}$ large glass spheres (Microspheres PSi-20.0, Kisker). The chamber containing the GUV is moved by a 3D piezo stage (Tritor $102 \mathrm{CAP}$, Piezosystem Jena) in $80 \mathrm{~nm}$ steps over the distance $y_{\mathrm{p}}$ relative to the trap center.

\subsection{Optical trapping and tracking}

As sketched in Fig. 1a, the displacement $\boldsymbol{b}=\left(b_{x}, b_{y}, b_{z}\right)$ of the particle from the trap centre is used to determine the underlying forces and energies during the induced uptake process.

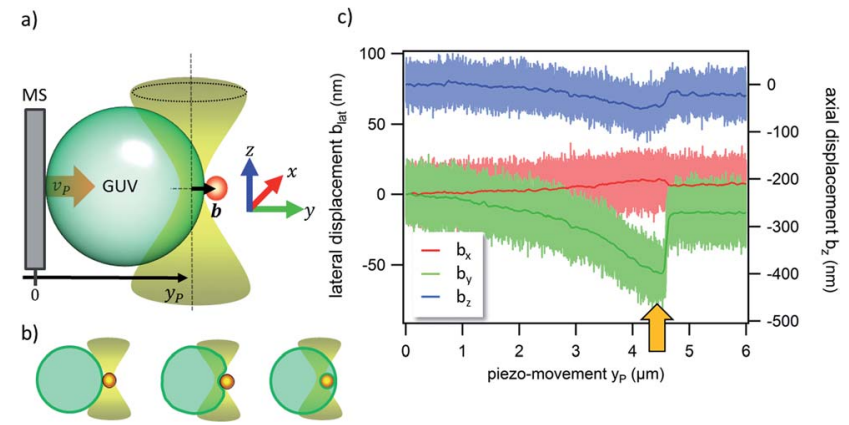

Fig. 1 (a) Sketch of the experimental procedure for the induced phagocytic particle uptake into a GUV fixed to a microstructure (MS). (b) Stepwise GUV penetration. (c) Displacement of the particle from the trap centre in the $x$-(red), $y$-(green) and $z$-direction (blue). The arrow indicates the uptake.

The optical force $F_{\mathrm{opt}, i}(i=x, y, z)$ shows a linear behaviour for lateral displacements $\left|b_{i}\right|<\lambda / 4$, where $\lambda=1064 \mathrm{~nm} / 1.33=800$ $\mathrm{nm}$ denotes the wavelength of the trapping laser light:

$$
F_{\mathrm{opt}, i}\left(b_{i}\right) \approx-\kappa_{i} b_{i}
$$

The trap stiffness $\kappa_{i}$ characterizes the strength of the optical trap in each direction and is determined by a Langevin calibration method. ${ }^{31}$

The corresponding optical energy $W_{\text {opt }, i}\left(b_{i}\right) \approx 1 / 2 \kappa_{i} b_{i}{ }^{2}+W_{i, 0}$ results in a harmonic potential.

The 3D particle tracking is realized by interference of the unscattered laser light and light scattered at the particle. The resulting interference intensity in the BFP $I\left(k_{x}, k_{y}, \boldsymbol{b}\right)$, which depends on the particle position $\boldsymbol{b}$, is recorded by using a QPD. The intensity profile $I\left(k_{x}, k_{y}, \boldsymbol{b}\right)$ is integrated over the four areas of the QPD, and provides four voltage signals $S_{n}(n=1, \ldots, 4)$. The linear composition of $S_{n}$ defines the position signals $S_{x}=$ $\left(\left(S_{1}+S_{3}\right)-\left(S_{2}+S_{4}\right)\right) / S_{0}, S_{y}=\left(\left(S_{1}+S_{2}\right)-\left(S_{3}+S_{4}\right)\right) / S_{0}$ and $S_{z}=$ $\left(S_{1}+S_{2}+S_{3}+S_{4}\right) / S_{0} . S_{0}$ is the sum signal without a scatterer. This operation is expressed by the function $Q_{i}$, which forms the signal triplet $S_{i}(i=x, y, z)$ encoding the 3D particle position:

$$
S_{i}\left(b_{i}\right)=\iint_{\mathrm{QPD}} I\left(k_{x}, k_{y}, \boldsymbol{b}\right) Q_{i} \mathrm{~d} k_{x} \mathrm{~d} k_{y} \approx S_{0, i}+g_{i} b_{i}
$$

The detector sensitivity $g_{i}$ relates the QPD signals $S_{i}$ to the particle displacement $b_{i}$. For small displacements, $\left|b_{i}\right|<\lambda / 4$ laterally and $\left|b_{i}\right|<\lambda / 2$ axially, this relationship is linear. The three sensitivities $g_{i}$ are obtained by the same Langevin calibration procedure in advance of each uptake experiment.

In the vicinity of the GUV, the interference pattern at the QPD may be altered by scattering of laser light at the GUV. This results in an additional intensity $\Delta I\left(k_{x}, k_{y}, d\right)$ and an additional sensitivity $\Delta g_{i}(d)$, which depends on the distance $d$ to the GUV: $S_{i}\left(b_{i}\right)=\iint(I(\boldsymbol{b})+\Delta I(d)) Q_{i} \mathrm{~d} k_{x y} \approx S_{0, i}+\left(g_{i}+\Delta g_{i}(d)\right) b_{i}$. It will be further discussed in Section 3.1. (for details, a manuscript is in preparation) that the change in sensitivity is small, i.e. $\left|\Delta g_{i}(d \rightarrow 0)\right|<0.1\left|g_{i}\right|$ leading to a relative position tracking error of less than $10 \%$. 


\subsection{Fluctuation analysis}

Due to the high photon densities available with the coherent BFP tracking technique, even particle fluctuations in the range of a few nanometres ( $\mathrm{nm}$ ) and on the scale of microseconds ( $\mu \mathrm{s})$ can be resolved. A common method in order to obtain relevant information about the Brownian motion of the particle is given by the autocorrelation (AC) function:

$$
\mathrm{AC}_{i}(\tau):=\left\langle b_{i}(t) b_{i}(t+\tau)\right\rangle=\left\langle b_{i}(0)^{2}\right\rangle \mathrm{e}^{-\tau / \tau_{0, i}}=k_{\mathrm{B}} T / \kappa_{i} \exp \left(-\tau \kappa_{i} / \gamma_{i}\right) .
$$

Here $\tau_{0, i}$ denotes the autocorrelation time, which can be obtained by an exponential fit to $\operatorname{AC}\left(b_{i}(t)\right)$. The zero-crossing of AC $\left(\tau_{i}=0\right)$ can be related to the stiffness $\kappa_{i}$ of the underlying potential by using the equipartition theorem $\left\langle b_{i}(0)^{2}\right\rangle=k_{\mathrm{B}} T / \kappa_{i}$. On a microsecond time scale, where $\tau \ll \tau_{0 i}$, the AC function in eqn (3) can be Taylor expanded to a linear dependency:

$$
\mathrm{AC}_{i}\left(\tau \ll \tau_{0 i}\right) \approx k_{\mathrm{B}} T\left(\frac{1}{\kappa_{i}}-\frac{\tau}{\gamma_{i}}\right)
$$

Applying a linear fit function to $\operatorname{AC}_{i}(\tau)$ for $\tau \leq 0.1 \tau_{0, i}$ allows one to determine the total stiffness $\kappa_{i}$ and the total friction coefficient $\gamma_{i}$ acting on the particle in all spatial directions. For the arrangement depicted in Fig. 1a, the two viscoelastic components are connected in parallel. Both the optical trap and the GUV-membrane represent damped oscillators with force constants $\kappa_{\mathrm{opt}}$ and $\kappa_{\mathrm{GUV}}$ and friction constants for the bead and GUV $\gamma_{\mathrm{b}}$ and $\gamma_{\mathrm{GUV}}$ such that

$$
\kappa_{i}=\kappa_{\mathrm{opt}, i}+\kappa_{\mathrm{GUV}, i} \quad \gamma_{i}=\gamma_{\mathrm{opt}, i}+\gamma_{\mathrm{GUV}, i}
$$

The fluctuation width $\Delta b_{i}$ or variance $\Delta b_{i}{ }^{2}=\left\langle b_{i}{ }^{2}\right\rangle-\left\langle b_{i}{ }^{2}\right\rangle$ can be obtained from the measured particle position histogram $p\left(b_{i}\right)$ from a piece of trajectory $b_{i}(t)$. After subtracting the mean value $\left\langle b_{i}\right\rangle$ the squared fluctuation width reads:

$$
\Delta b_{i}{ }^{2}=\int b_{i}{ }^{2} p_{0} \exp \left(-W_{\text {tot }}\left(b_{i}\right) / k_{\mathrm{B}} T\right) \mathrm{d} b_{i}
$$

This expression is valid for all shapes of $p\left(b_{i}\right)=$ $p_{0} \exp \left(-\left(W_{\mathrm{opt}}\left(b_{i}\right)+W_{\mathrm{GUV}}\left(b_{i}, d\right)\right) / k_{\mathrm{B}} T\right) \mathrm{d} b_{i}$, which obey Boltzmann statistics. Generally, it is defined by the harmonic trapping potential $W_{\text {opt }}\left(b_{i}\right)$ as well as by the distance dependent potential $W_{\mathrm{GUV}}\left(b_{i}, d\right)$ generated by the presence of the GUV. In summary, the particle fluctuations can be characterised by the three fluctuation variables $\kappa, \gamma$ and $\Delta b$, which all provide relevant information about the local environment of the particle.

\subsection{Deformation model}

The induced phagocytic particle uptake is accompanied by a successive deformation of the GUV until the engulfment is finally completed. This process can be mathematically described by a simple energy model considering the behaviour of the particle-GUV complex. The required deformation energy $G_{\text {def }}$ of a lipid bilayer sheet with membrane tension $\sigma$ is based on the Helfrich energy, ${ }^{32}$ which, neglecting Gaussian and spontaneous curvature effects, can be expressed as:

$$
G_{\mathrm{def}}=\int_{A_{g}}\left(\frac{K}{2}(2 H)^{2}+\sigma\right) \mathrm{d} x \mathrm{~d} y=G_{\mathrm{ben}}+G_{\text {str }}
$$

here, the first term corresponds to the bending energy $G_{\text {ben }}$ with the bending modulus $K$ and the second term represents the stretching energy $G_{\text {str }}$. The contribution of adhesion energy is neglected in the following since it is not relevant to the utilized particle-vesicle system in our experiment (see Section 3.2). The membrane curvature $2 H(x, y)=1 / R_{1}(x, y)+1 / R_{2}(x, y)$ can be expressed by the two principal radii of curvatures $R_{1}$ and $R_{2}$. If the GUV with radius $R_{\mathrm{g}}$ is spherical, then $2 H_{\mathrm{sp}}(x, y)=2 / R_{\mathrm{g}}$. In general, however, $G_{\text {def }}$ has to be integrated over the arbitrarily deformed surface $A_{g}$ of the GUV.

As sketched in Fig. 2a, the deformation of the GUV is comprised of a global (gl) and a local (lo) deformation:

$$
G_{\mathrm{def}}\left(h_{\mathrm{gl}}, h_{\mathrm{lo}}\right)=G_{\mathrm{def}}^{\mathrm{gl}}\left(h_{\mathrm{gl}}\right)+G_{\mathrm{def}}^{\mathrm{lo}}\left(h_{\mathrm{lo}}\right)
$$

This energy increases with a global and a local indentation height, $h_{\mathrm{gl}}$ and $h_{\mathrm{lo}}$, which are variables for the GUV deformation.

2.6.1. Global deformation energy. The global shape of the spherical vesicle is transformed into an oblate ellipsoid characterized by the two half-axes $a$ and $c<a$ (see Fig 2a). Whereas the short half-axis $c:=R_{\mathrm{g}}-h_{\mathrm{gl}}$ is reduced by the global indentation height $h_{\mathrm{gl}}$, the long axis $a$ is increased according to

$$
a=R_{\mathrm{g}} \sqrt{R_{\mathrm{g}} /\left(R_{\mathrm{g}}-h_{\mathrm{gl}}\right)}
$$

Eqn (9) is derived from the assumption that the GUV volume is conserved during the deformation process, i.e. $R_{\mathrm{g}}{ }^{3}=a^{2} c$.

The area of the ellipsoid is $A_{\text {ell }}=2 \pi a^{2}\left(1+(c / a)^{2}(\tan h \varepsilon / \varepsilon)\right)$, where $\varepsilon=\sqrt{1-(c / a)^{2}}$ denotes the eccentricity. The increase in surface area is $\Delta A_{\mathrm{gl}}\left(h_{\mathrm{gl}}\right)=A_{\mathrm{ell}}\left(h_{\mathrm{gl}}\right)-A_{\mathrm{sp}}\left(h_{\mathrm{gl}}\right)$, with sphere surface $A_{\mathrm{sp}}=4 \pi R_{\mathrm{g}}{ }^{2}$.

The global deformation energy $G_{\mathrm{def}}^{\mathrm{gl}}$ can be further separated into components for stretching and bending:

$$
G_{\mathrm{def}}^{\mathrm{gl}}\left(h_{\mathrm{gl}}\right)=G_{\mathrm{str}}^{\mathrm{gl}}\left(h_{\mathrm{gl}}\right)+G_{\mathrm{ben}}^{\mathrm{gl}}\left(h_{\mathrm{gl}}\right)
$$
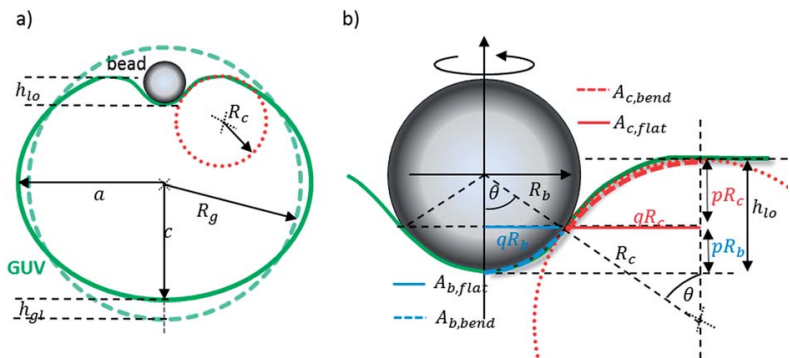

Fig. 2 Geometry used to model the deformation energy of the particle-GUV complex. (a) The particle induces a global and local deformation of the GUV profile. The global indentation is described by $h_{\mathrm{gl}}$. (b) Local deformation: the particle gets wrapped by a spherical cap with area $A_{\text {int. }}$. The height of the cap is $p R_{\mathrm{b}}$ and the height of circle segment is $p R_{\mathrm{c}}$. The local indentation height is described by $h_{\mathrm{lo}}=$ $p\left(R_{\mathrm{b}}+R_{\mathrm{c}}\right)=(1-\cos (\theta))\left(R_{\mathrm{b}}+R_{\mathrm{c}}\right)$. 
Using the mean curvature of an oblate ellipsoid $2 H_{\text {ell }}\left(h_{\mathrm{gl}}\right)=$ $\left(c / a^{2}+a / c^{2}\right)$ the global bending energy with bending modulus $K$ is

$G_{\mathrm{ben}}^{\mathrm{gl}}\left(h_{\mathrm{gl}}\right)=G_{\mathrm{ben}}^{\mathrm{ell}}\left(h_{\mathrm{gl}}\right)-G_{\mathrm{ben}}^{\mathrm{sp}}\left(h_{\mathrm{gl}}\right)=K / 2\left[\left(2 H_{\mathrm{ell}}\right)^{2} A_{\mathrm{ell}}-\left(2 H_{\mathrm{sp}}\right)^{2} A_{\mathrm{sp}}\right]$

The global stretching energy, which is typically much smaller than the global bending energy, is proportional to the increase in surface area,

$$
G_{\mathrm{str}}^{\mathrm{gl}}\left(h_{g l}\right)=\sigma \Delta A_{\mathrm{gl}}\left(h_{\mathrm{gl}}\right)
$$

2.6.2. Local deformation energy. As pointed out further in Fig. 2b, the local deformation energy

$$
G_{\mathrm{def}}^{\mathrm{lo}}\left(h_{\mathrm{lo}}\right)=G_{\mathrm{str}}^{\mathrm{lo}}\left(h_{\mathrm{lo}}\right)+G_{\mathrm{ben}}^{\mathrm{lo}}\left(h_{\mathrm{lo}}\right)
$$

increases with the local indentation height $h_{\mathrm{lo}}$. It can be separated into a convex spherical indentation with bead radius $R_{\mathrm{b}}$ and a concave toroidal indentation with radii $R_{\mathrm{c}}$ and $q\left(R_{\mathrm{b}}+R_{\mathrm{c}}\right)$ where $q=\sin (\theta) \cdot{ }^{33,34}$ We are interested in the increase in surface areas and in total curvature from a flat membrane to a bended membrane.

Therefore we use the wrapping height of the bead $p R_{\mathrm{b}}, p(\theta)=$ $1-\cos (\theta)$, and of the circle segment with wrapping height $p R_{\mathrm{c}}$, which defines the local indentation height

$$
h_{\mathrm{lo}}=p\left(R_{\mathrm{b}}+R_{\mathrm{c}}\right)=(1-\cos (\theta))\left(R_{\mathrm{b}}+R_{\mathrm{c}}\right)
$$

$R_{\mathrm{b}}+R_{\mathrm{c}}$ represents the distance of the circle centres, and $\theta$ is the angle to the normal. $q\left(R_{\mathrm{b}}+R_{\mathrm{c}}\right)$ is the normal projection and defines the flat circular area $A_{\mathrm{b} \text {,flat }}+A_{\mathrm{c} \text {,flat }}=q^{2}\left(R_{\mathrm{b}}+R_{\mathrm{c}}\right)^{2} \pi$ that increases to $A_{\mathrm{b} \text {,bend }}+A_{\mathrm{c} \text {,bend }} \cdot A_{\mathrm{b} \text {,flat }}=q^{2} R_{\mathrm{b}}{ }^{2} \pi$ is a circular area, and $A_{\mathrm{c}, \text { flat }}=q^{2}\left(\left(R_{\mathrm{b}}+R_{\mathrm{c}}\right)^{2}-R_{\mathrm{b}}{ }^{2}\right) \pi$ is the area of the surrounding ring.

The increase in local surface area due to the deformation with an indenting sphere of radius $R_{\mathrm{b}}$ is $\Delta A_{\mathrm{sph}} \approx A_{\mathrm{b} \text {,bend }}-$ $A_{\mathrm{b}, \text { flat }}=\pi\left(p R_{\mathrm{b}}\right)^{2}$. The increase in local area due to the toroidal deformation is $\Delta A_{\text {tor }} \approx A_{\text {c,bend }}-A_{\text {c,flat }}=\pi q R_{\mathrm{c}}{ }^{2}(q+2)$. The surface area of a torus between the polar angles $[0 ; \theta]$ is $A_{\text {tor }}=2 \pi R_{\mathrm{c}}{ }^{2} \sin (\theta)+2 \pi R_{\mathrm{c}} R_{\mathrm{t}} \theta \approx 2 \pi R_{\mathrm{c}} q\left(R_{\mathrm{c}}+R_{\mathrm{t}}\right)$ if $\theta<\pi / 4$. Here, $R_{\mathrm{c}}$ is the radius of the tube, and $R_{\mathrm{t}}$ is the radius of the torus ring. From eqn (14) we find $p=h_{\mathrm{lo}} /\left(R_{\mathrm{b}}+R_{\mathrm{c}}\right)$ and $q=\sqrt{2 h_{\mathrm{lo}} /\left(R_{\mathrm{b}}+R_{\mathrm{c}}\right)}$, which can be inserted into $\Delta A_{\mathrm{tor}}(\theta)$ and $\Delta A_{\mathrm{sph}}(\theta)$ to obtain the local stretching energy as a function of $h_{\mathrm{lo}}$ and $R_{\mathrm{bc}}=R_{\mathrm{b}}+R_{\mathrm{c}}$

$$
\begin{aligned}
G_{\mathrm{str}}^{\mathrm{lo}}\left(h_{\mathrm{lo}}\right) & =\sigma \Delta A_{\mathrm{sph}}\left(h_{\mathrm{lo}}\right)+\sigma \Delta A_{\mathrm{tor}}\left(h_{\mathrm{lo}}\right) \\
& =\sigma \pi\left(\frac{h_{\mathrm{lo}} R_{\mathrm{b}}}{R_{\mathrm{bc}}}\right)^{2}+\sigma \pi R_{\mathrm{c}}{ }^{2}\left(\frac{2 h_{\mathrm{lo}}}{R_{\mathrm{bc}}}+2 \sqrt{\frac{2 h_{\mathrm{lo}}}{R_{\mathrm{bc}}}}\right) .
\end{aligned}
$$

The local bending energy produced by the indentation of the bead is defined by the local curvature $2 / R_{\mathrm{b}}$ and the area $A_{\mathrm{b}, \text { bend }}=2 \pi R_{\mathrm{b}}{ }^{2} p$ of the cap with height $p R_{\mathrm{b}}: G_{\mathrm{ben}}^{\mathrm{sph}}(\theta)=$ $K / 2\left(2 / R_{\mathrm{b}}\right)^{2} A_{\mathrm{b}, \text { bend }}=4 \pi K p$.
Similar to the approach of Deserno (eqn (31) in ref. 33), we calculate the curvature energy in the torus segment with opening angle $\theta$. The energy as a function of the wrapping angle is simplified to $G_{\text {ben }}^{\mathrm{tor}}(\theta)=K / 2 \times 2 \pi \int_{0}^{\theta}\left(1 / R_{\mathrm{c}}+1 / R_{\mathrm{b}}\right)^{2} \times q R_{\mathrm{bc}} \times$ $\left(q R_{\mathrm{bc}} \cos \left(\theta^{\prime}\right)+R_{\mathrm{c}}\right) \mathrm{d} \theta^{\prime}=K \pi\left(1 / R_{\mathrm{c}}+1 / R_{\mathrm{b}}\right)^{2}\left(R_{\mathrm{bc}}{ }^{2} q^{3 / 2} / 3+p R_{\mathrm{bc}} R_{\mathrm{c}}\right)$.

Therefore, we estimate the local bending energy to be

$$
\begin{aligned}
G_{\mathrm{ben}}^{\mathrm{lo}}\left(h_{\mathrm{lo}}\right)= & K \pi \frac{4 h_{\mathrm{lo}}}{R_{\mathrm{bc}}}+K \pi\left(\frac{1}{R_{\mathrm{c}}}+\frac{1}{R_{\mathrm{b}}}\right)^{2} \\
& \times\left(\sqrt{R_{\mathrm{bc}}}\left(2 h_{\mathrm{lo}}\right)^{3 / 2} / 3+2 h_{\mathrm{lo}} R_{\mathrm{c}} \cdot R_{\mathrm{c}}\right) .
\end{aligned}
$$

The local and global deformation forces act in parallel, leading to the effect that the weaker global indentation distance is larger than the local one, such that $h_{\text {lo }}=Z h_{\mathrm{gl}}$. Here, we approximate $Z$ as the ratio $Z=G_{\mathrm{def}}^{\mathrm{gl}}\left(h_{0}\right) / G_{\mathrm{def}}^{\mathrm{lo}}\left(h_{0}\right)$ of global and local deformation energies at a distinct distance, e.g. $h_{0}=1 \mu \mathrm{m}$. Our approach assumes quadratic potentials $G_{\text {def }}(h)$ and corresponds to the inverse addition of spring constants for serially coupled oscillators. In consequence we can write:

$$
G_{\mathrm{def}}(h)=G_{\mathrm{ben}}^{\mathrm{lo}}(Z h)+G_{\mathrm{str}}^{\mathrm{lo}}(Z h)+G_{\mathrm{ben}}^{\mathrm{glo}}(h)+G_{\mathrm{str}}^{\mathrm{glo}}(h)
$$

\section{Experimental results}

\subsection{Influence of the GUV on bead trapping and tracking}

Before facing the induced phagocytic uptake, the influence of the GUV membrane (as an additional scatterer) on particle trapping and BFP tracking has to be verified and discussed. Therefore we investigated the detector response of a single GUV using the experimental configuration sketched in Fig. 3a. ${ }^{35,36}$ The location dependent detector response for a freely diffusing GUV with radius $R_{\mathrm{g}} \approx 9.4 \mu \mathrm{m}$ (measured by fluorescence microscopy) was recorded while performing a fast periodic displacement of the laser focus $y_{\mathrm{f}}$ with $f_{\mathrm{t}}=400 \mathrm{~Hz}$ along the $y$-direction passing both vesicle edges $y_{\text {le }}$ and $y_{\text {re }}$. The movement of the laser focus over the trap length of $L_{\mathrm{t}}=28 \mu \mathrm{m}$ is achieved using galvanometric scan mirrors.

The QPD-signal for the $y$-direction as a function of the focus position $y_{\mathrm{f}}$ is shown in Fig. 3b for a constant laser power. An asymmetric response $S_{\mathrm{g}}(y)$ within a range of about $3 \mu \mathrm{m}$ of the vesicle edges is shaded in bright green. ${ }^{37}$ As indicated, the distance between the zero-crossings can be used quite well to determine the vesicle radius $R_{\mathrm{g}}$, which can also be read out from video microscopy. Within the vesicle $\left(y_{\text {le }}<y_{\mathrm{f}}<y_{\text {re }}\right)$, the focus positions lead to a vanishing influence on the detector response (shaded in green). If the focus is far enough from the vesicle edge, the QPD-signal drops to zero again (grey shaded area).

The plot in Fig. 3c presents the detector responses in the vicinity of the right vesicle edge $y_{\text {re }}$ for a linearly varied laser power from 10-50 $\mathrm{mW}$ measured at a reference diode. The displacement $b_{y}=y_{\mathrm{f}}-y_{0, \mathrm{r}}$ describes the distance of the focus 
a)

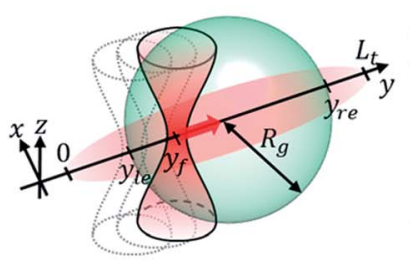

b)

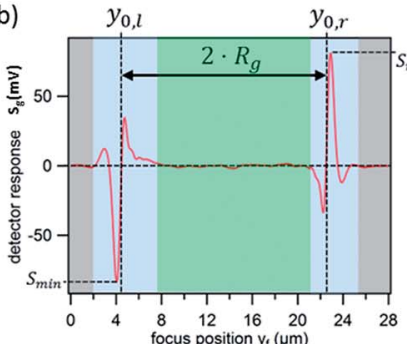

c)

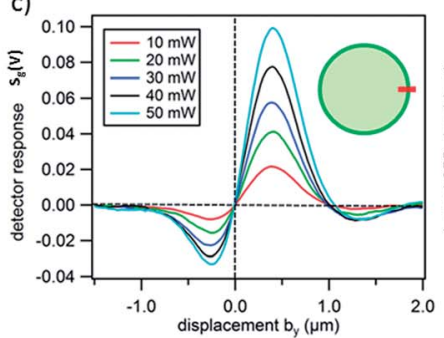

d)

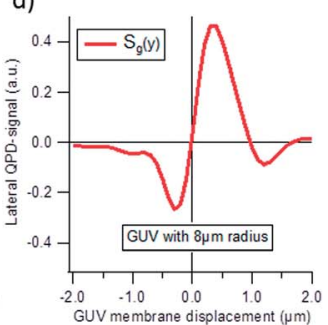

Fig. 3 Influence of the GUV on the BFP tracking of the particle. (a) A sweeping laser focus determines the scattering signal from the GUV. (b) Interferometric detector responses $S_{g}(y)$ from the edges of the GUV obtained by a complete focus scan of length $L_{t}=28 \mu \mathrm{m}$. (c) Detector responses $S_{g}(y)$ from the right edge of the GUV membrane for different laser powers. The signals $S_{g}(y)$ do not shift laterally. (d) $\operatorname{Signal} S_{g}(y)$ obtained from wave optical simulation.

position to the corresponding central zero-crossing of the detector response. The results clearly show that the shape and position of the signals $S_{\mathrm{g}}(y)$ do not change or shift laterally, although the laser power and thereby the optical forces are increased. In consequence, the optical forces on the GUV are negligible.

In the experiment, the position of the vesicle edge within the response function cannot be directly inferred. Therefore we performed a numerical simulation, ${ }^{38}$ where the interference of focussed unscattered light and light scattered at a GUV $\left(R_{\mathrm{g}}=8 \mu \mathrm{m}\right)$ is recorded and read out by a QPD. Fig. 3d shows that the edge of the GUV corresponds to the zero-crossing of the signal $S_{\mathrm{g}}(y)$ at $y_{0, \mathrm{r}}$.

The influence of the light scattered at the GUV on the signal of the trapped $1 \mu \mathrm{m}$ polystyrene bead is relatively small. As shown in Fig. $3 \mathrm{~b}-\mathrm{c}$, the maximum amplitude of the signal, $S_{\max }$, at a laser power $P=50 \mathrm{~mW}$ is $0.1 \mathrm{~V}$, whereas the maximum signal of the bead is $1.8 \mathrm{~V}$. Although the scattered electric fields have to be added and not the interference intensities, the approximation to add simply the QPD signals is reasonable and helpful. $^{35,39}$ Based on this approach, the change in position signal $\Delta I(d)$, in detector sensitivity $\Delta g(d)$ and in position $\delta b$ is less than $0.1 \mathrm{~V} / 1.8 \mathrm{~V}=6 \%$. In summary, light scattering at the GUV has a negligible influence on both optical forces and position tracking of the particle.

\subsection{Process of contacting the GUV}

The study of a phagocytic particle uptake using a biomimetic system is initiated by the contact process between the GUV and the particle. Here, the particle position fluctuations in the vicinity of the GUV, measured at $1 \mathrm{MHz}$ in all three directions, allow for the interpretation of the interaction process.

The experimental procedure is shown in Fig. 4a. Here the unfixed GUV moves with the piezo-stage (PS) and is approached in $20 \mathrm{~nm}$ steps towards the trapped particle. At each position, $2 \times 10^{5}$ particle positions are recorded over $200 \mathrm{~ms}$. Assuming thermal equilibrium, the fluctuation width $\Delta b_{i}(i=x, y, z)$ is a useful variable to characterize the interaction process. The plot in Fig. $4 \mathrm{~b}$ reveals a slight decrease of the fluctuation width of the radial trajectory $b_{y}\left(y_{\mathrm{p}}=v_{\mathrm{p}} t\right)$ upon contact at $d=0$. It should be noted that the mean value of the displacements $\left\langle b_{y}\right\rangle$ (central red line) does not change more than $\pm 5 \mathrm{~nm}$ upon contact with the unfixed GUV.
However, this is different with the lateral and axial friction factors $\gamma_{i}(d)$ and force constants $\kappa_{i}(d)$, which increase with decreasing distance $d$ to the GUV (see Fig. $4 \mathrm{c}$ and d). $\gamma_{i}$ and $\kappa_{i}$ were obtained by linear fits to the AC-functions as described by eqn (4). An optimization procedure revealed that the most reliable results for linear fits were obtained for fitting intervals of $35 \mu$ s corresponding to 35 points in the AC data curve recorded for each piezo position $y_{\mathrm{p}}$. The total force constants (stiffness) were obtained through the constant part of the linear fit curve, $\kappa=k_{\mathrm{B}} T / \mathrm{AC}(0)$, whereas the friction coefficients were obtained from the slope of the linear fit curve. In addition, $\kappa$ was positively cross-checked through evaluating the fluctuation width $\Delta b$, thus $\kappa=k_{\mathrm{B}} T / \Delta b^{2}$.

The most important effect visible in Fig. $4 \mathrm{c}$ is the smooth increase in $\kappa_{y}$ in the radial $y$-direction, indicating that no binding of the particle to the GUV-membrane occurs. A particle
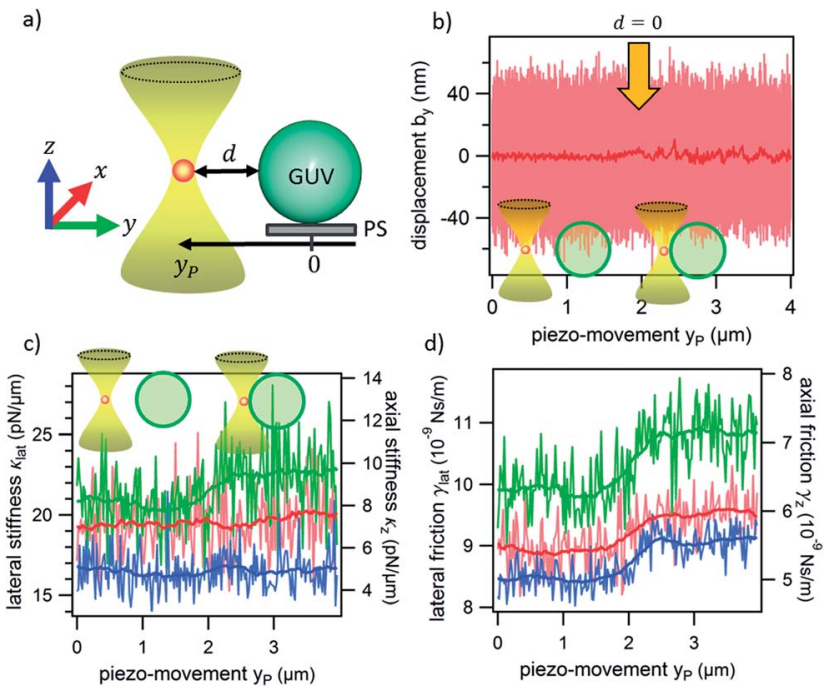

Fig. 4 GUV-particle contact process: (a) scheme for approaching the unfixed GUV to the trapped particle by a stepwise piezo-movement. (b) Displacement of the particle in the $y$-direction. The solid red curve represents the mean value over $10^{6}$ samples. The arrow indicates the contact process. (c) Change of the force constants $\kappa_{i}$ in the $x$ - (red), $y$ - (green) and $z$-direction (blue) during the contact process. (d) Change of the friction constants $\gamma_{i}$ in the $x$ - (red), $y$ - (green) and $z$-direction (blue) during the contact process. 
binding process to an interface is characterized by an abrupt increase in $\kappa^{27,40}$ due to a sudden decrease in the fluctuation width of the particle. Therefore adhesion energies are not considered in our energy model as introduced in Section 2.6. Whereas the fluctuation width and thereby the force constants hardly change in tangential directions $x$ and $z$, the friction experienced by the particle increases in all three directions. Remarkably, the friction factor $\gamma$ and correspondingly the diffusion constant $k T / \gamma$ change already prior to binding, and thus indicate a hydrodynamic coupling between the particle and GUV membrane. ${ }^{40}$ For $y_{\mathrm{p}}>2 \mu \mathrm{m}$, the bead is in closer contact with the GUV (the collision rate is increased) and the bead diffusion is influenced by the higher viscosity close to the lipid bilayer. Similar to the increase in $\gamma$ close to a stiff wall, the increase in radial $y$-direction is larger than in tangential $x, z$ directions. This method may even allow distinguishing between different viscosities of the membrane due to different molecular compositions.

\subsection{Induced phagocytosis}

In this paragraph the phagocytic particle uptake induced by the optical trap is introduced. In the experiment, the uptake process is characterized by two parameters of the GUV: the vesicle radius $R_{\mathrm{g}}$ and the membrane tension $\sigma$. Both values are not known in advance, but methods in order to determine them are provided in the following.
3.3.1. Uptake force and energy profiles. The PFM setup ${ }^{31}$ allows the realization of induced phagocytic particle uptake while tracking the particle in 3D and observing the GUV-particle complex via fluorescence or brightfield microscopy. The extent of the GUV deformation can be extracted from the fluorescent images of the GUV-particle complex that are recorded for each piezo-position $y_{\mathrm{p}}$. In Fig. 5a selected images from a video sequence of complete particle uptake are presented. For contrast enhancement, a Laplace filter has been applied to each single frame. The dot-like particle gets gradually engulfed by the GUV membrane until the particle pinches off into the intravesicular space. The deformation of the GUV appears in two different ways. First, it emerges on a global scale transforming the shape into a horizontally compressed and vertically stretched (oblate) ellipsoid. Second, an enforced wrapping process can locally be observed in the surrounding of the particle. The degree of global (elliptical) deformation is quantified by fitting ellipses to the edges of the GUV images in Fig. 5a. The resulting change in the length of both half-axes is plotted in Fig. 5b and is in good agreement with the expected theoretical behaviour described by eqn (9).

The 3D-tracking of the particle displacement from the trap centre allows for the determination of the 3D optical forces by integration of the potentials (see Section 2.4). The uptake force is dominated by the radial $y$-component, whereas the tangential directions make up a significantly smaller but still resolvable amount (not shown). a)

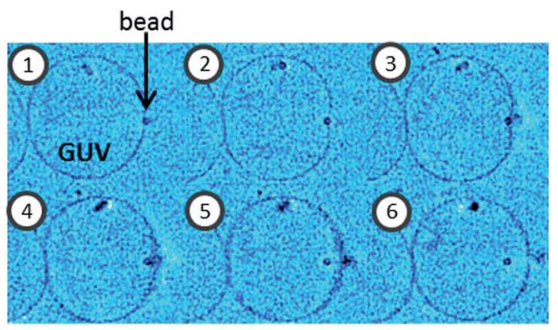

d)

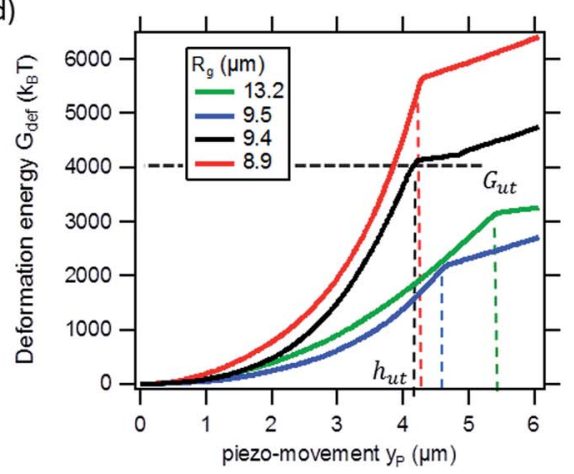

b)

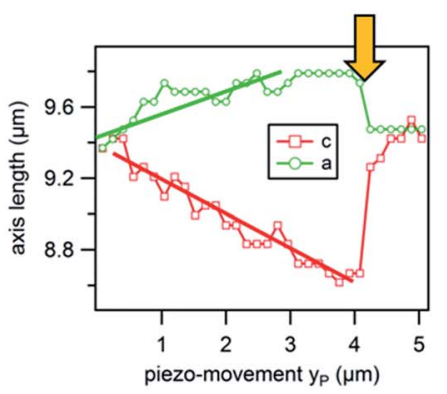

e)

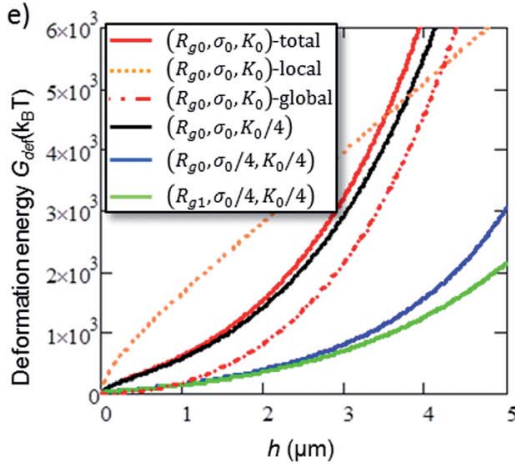

c)

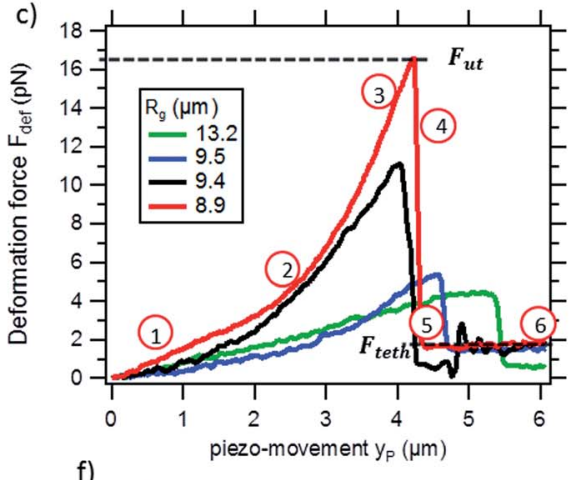

f)

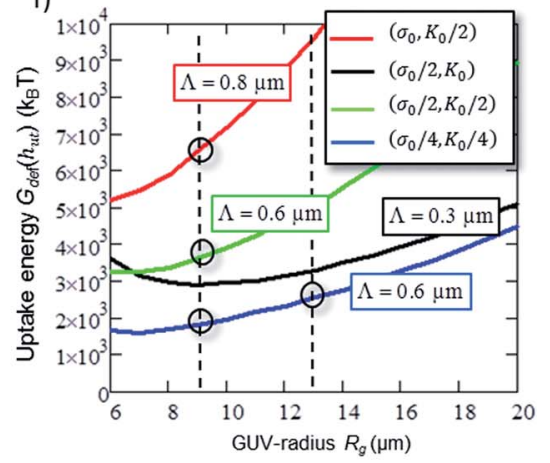

Fig. 5 Complete induced phagocytic particle uptake. (a) Time series of fluorescent images demonstrating the deformation of the GUV. (b) Change in the half-axes of the ellipsoidal GUV extracted from Fig. 5a. (c) Measured deformation force as a function of the piezo-movement. The numbers correspond to the images in Fig. $5 a$. $F_{\text {teth }}$ is the tether force. (d) Measured deformation energy profiles for different GUVs. (e) Simulated deformation energy profiles as a function of the indentation height $h$ for GUVs with $R_{\mathrm{go}}=9 \mu \mathrm{m}, R_{\mathrm{g} 1}=13 \mu \mathrm{m}, K_{0}=10 k_{\mathrm{B}} T, \sigma_{0}=30 k_{\mathrm{B}} T \mu \mathrm{m}^{-2}=$ $0.12 \times 10^{-3} \mathrm{pN} \mathrm{nm}{ }^{-1}$ and $R_{\mathrm{c}}=K_{0} / 10 \mu \mathrm{m} / k_{\mathrm{B}} T$. (f) Simulated uptake energies at uptake lengths $h_{\mathrm{ut}}=1+R_{\mathrm{g}} / 3$ for different GUV radii $R_{\mathrm{g}}$ and $\Lambda$. 
The deformation force profiles $F_{\text {def }}\left(y_{\mathrm{p}}\right)$ for four different vesicles shown in Fig. $5 \mathrm{c}$ in dependence of the piezo-position $y_{\mathrm{p}}$ reveals a nonlinear behaviour corresponding to the frames (1)(3) until the internalization process is completed at the uptake force $F_{\text {ut }}$. Subsequently, the force drops off in the vicinity of frame (4), which corresponds to a relaxation process of the particle towards the trap centre. In frames (5) and (6) the particle reaches the equilibrium position accompanied by a non-vanishing force. Although badly visible in Fig. 5a, the remaining force likely belongs to a membrane tether indicating that the particle is not completely disrupted from the membrane and a tether is formed. The constant tether force is about $F_{\text {teth }} \approx 1.7 \mathrm{pN}$ and can be calculated by $F_{\text {teth }}=8 \pi \sqrt{K \sigma}$ for a known membrane tension and bending modulus. ${ }^{41}$

In Fig. $5 \mathrm{c}$ and $\mathrm{d}$ the measured profiles of the required forces and energies for complete particle uptake (ut) with maximum values $G_{\mathrm{ut}}$ and $F_{\mathrm{ut}}$ are displayed for different vesicle radii $R_{\mathrm{g}}$. Vesicle sizes are determined by video microscopy. As illustrated, the required deformation energies for complete uptake vary between $G_{\mathrm{ut}}=2000-5500 k_{\mathrm{B}} T$, whereas the forces range between $F_{\text {ut }}=4-16 \mathrm{pN}\left(k_{\mathrm{B}} T=4 \mathrm{pN} \mathrm{nm}\right.$ at $\left.T=300 \mathrm{~K}\right)$. The required indentation height (piezo-movement $y_{\mathrm{p}}$ ) for the complete particle ingestion, the uptake length $h_{\mathrm{ut}}$, is between $4.2 \mu \mathrm{m}$ and $5.5 \mu \mathrm{m}$.

Results from the model. In Fig. 5d we have used the energy model introduced in Section 2.6 to prove its correctness and compare it with the experimental results. Fig. 5e shows four simulated energy profiles for different GUVs, where one profile (red curve) is separated into the global (red dash-dotted line) and local (orange dotted line) component of the total GUV deformation. The curves cannot be added on the same length scale $h$ since $h_{\mathrm{lo}}=Z h_{\mathrm{gl}}$ where $Z=0.1$ in this example (see eqn (17)). We used the same radii $R_{\mathrm{g} 0}=9 \mu \mathrm{m}$ and $R_{\mathrm{g} 1}=13 \mu \mathrm{m}$ as in the experiment. The membrane tension was assumed to be $\sigma_{0}=$

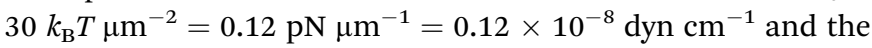
bending modulus $K_{0}=10 k_{\mathrm{B}} T,{ }^{28}$ such that $F_{\text {teth }}=8 \pi(K \sigma)^{1 / 2}=1.7$ $\mathrm{pN}$, which corresponds to the measured tether force. The radii for the two membrane curvatures for the local deformation were $R_{\mathrm{b}}=0.5 \mu \mathrm{m}$ for the bead and initially $R_{\mathrm{c}}=2.5 \mu \mathrm{m}$ for the torus tube. We than adapted the radius of curvature $R_{\mathrm{c}}$ to be proportional to $K$, such that $R_{\mathrm{c}}=K / 10 \mu \mathrm{m} / k_{\mathrm{B}} T$. However, the effect of $R_{\mathrm{c}}$ on $G_{\mathrm{def}}(h)$ was rather small. For the above mentioned parameters, deformation energies of up to $5500 k_{\mathrm{B}} T$ are reached for a local indentation $h_{\mathrm{lo}}$ of up to $1 \mu \mathrm{m}$ and a global indentation $h_{\mathrm{g} 1}$ of also up to $3 \mu \mathrm{m}$, resulting in a total indentation $h_{\mathrm{lo}}+h_{\mathrm{g} 1}$ of up to $4 \mu \mathrm{m}$. According to our model the main energy contribution for small indentations $(h<1 \mu \mathrm{m})$ results from the local deformation of the GUV. By changing the ratio $\Lambda^{2}=(K / \sigma)$ between the bending modulus and the membrane tension, different local slopes of the energy curves can be obtained. The black curve displays a $4 \times$ decrease in $\sigma$, which results in an only slightly smaller energy profile, whereas a $4 \times$ decrease in $K$ reduced the deformation energy significantly. In our case $\left(\sigma_{0} \approx\right.$ $30 k_{\mathrm{B}} T \mu \mathrm{m}^{-2}$ and $K_{0}=10 k_{\mathrm{B}} T$ ) the bending rigidity influences the deformation energy profile much less than the membrane tension. A $4 \times$ decrease of the bending tension $\sigma_{0}$ decreases the energy at $h=4 \mu \mathrm{m}$ significantly. The $40 \%$ increase in GUV size
$\left(R_{\mathrm{g} 0} \rightarrow R_{\mathrm{g} 1}\right)$ leads to a $40 \%$ reduced deformation energy at $h=4.5 \mu \mathrm{m}$. From Fig. 5e we can estimate the uptake length $h_{\mathrm{ut}}$ to be about one third of the vesicle radius plus the diameter of the bead, such that $G_{\mathrm{def}}\left(h_{\mathrm{ut}}\right)$ was assumed as the uptake energy $G_{\mathrm{ut}}$ in our model. The uptake length is the sum of the local and global indentation, $h_{\mathrm{ut}}=h_{\mathrm{lo}}+h_{\mathrm{gl}}=R_{\mathrm{g}} / 3+2 R_{\mathrm{b}}$, where the particle is taken up and the free energy drops down. In Fig. $5 \mathrm{f}$ the overall increase of $G_{\mathrm{ut}}$ with the vesicle radius is displayed for different ratios of $\Lambda=\operatorname{sqrt}(K / \sigma)=0.3,0.5$ and 0.9 , which are all values for vesicles with the mean stiffness. For vesicles with a decreased bending modulus $\Lambda=0.3$, the uptake energy rises strongly with the vesicle radius. Uptake energies and vesicle radii found in the experiment are marked by black circles. Generally, rupturing and a possible tether formation are not included in our model.

3.2.2. Elastic and viscous behaviour during uptake. Similar to the idea presented in Section 3.2, where we measured and analysed the particle fluctuations prior to contact with the GUV, we analyse now the change in fluctuations during the wrapping process. The analysis procedure is described in Section 2.5. Fig. 6a illustrates the elastic behaviour of the bead, which is characterized in all three directions by the change in stiffness $\kappa_{i}\left(y_{\mathrm{p}}\right)$ (see eqn (5)) as a function of the indentation height $h \sim y_{\mathrm{p}}$ into the membrane of a fixed GUV. In Fig. $6 \mathrm{~b}$ the viscous behaviour characterized by the friction coefficient $\gamma_{i}\left(y_{\mathrm{p}}\right)$ is displayed $(i=x, y, z)$. The course of the fluctuation width $\Delta b_{i}\left(y_{\mathrm{p}}\right)$ is consistent with the results of the corresponding stiffness $\kappa_{i}$ (not shown). The stiffness $\kappa_{z}$ in the $z$-direction remains approximately constant for all $y_{\mathrm{p}}$, i.e. $\Delta \kappa_{z} \approx 0$, which means that the axial width of the fluctuations is not reduced by the membrane. However, in the other two directions, a smooth increase of tangential stiffness $\Delta \kappa_{x}=\kappa_{x}-\kappa_{\mathrm{opt}, x}$ to $\kappa_{\mathrm{m}} \approx 40 \mathrm{pN} \mu \mathrm{m}^{-1}$ at $h_{\mathrm{lo}} \approx 2 R_{\mathrm{b}}$ and a steeper increase of the radial stiffness $\Delta \kappa_{y}$ to $\kappa_{\mathrm{m}}$ can be observed.

Whereas the radial fluctuations are dominated by the membrane fluctuations and thereby define the steeper stiffness change $\Delta \kappa_{y}$, the reduced fluctuation volume of the trapped bead leads to a linear increase of $\Delta \kappa_{x}$, over the first micrometres. Only for a larger indentation of the bead at $h_{\mathrm{lo}} \approx 2 R_{\mathrm{b}}, \kappa_{x}$ also approaches the value of $\kappa_{\mathrm{m}}$.
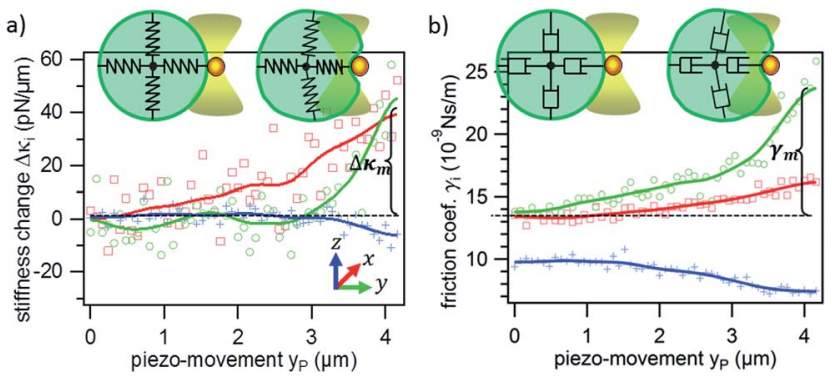

Fig. 6 Analysis of particle fluctuations after contact, i.e. during the induced uptake process. Lines are mean values to the measured data (markers). (a) Stiffness change in the $x$-(red), $y$-(green) and $z$-direction (blue) for increasing indentation. (b) The three friction coefficients for increasing indentation using the identical color encoding. 
The changes in viscous drag are relatively small in the two tangential directions, i.e. $\Delta \gamma_{x z}\left(y_{\mathrm{p}}\right) / \Delta \gamma_{x z}\left(y_{\mathrm{p}}=4 \mu \mathrm{m}\right)<0.25$, as shown in Fig. 6b. The increase in radial friction is more pronounced: $\Delta \gamma_{y}$ grows smoothly until $y_{\mathrm{p}} \leq 3 \mu \mathrm{m}$ and much stronger for $y_{\mathrm{p}}>3 \mu \mathrm{m}$ until the maximal value of $\Delta \gamma_{y}\left(h_{\mathrm{lo}} \approx 2 R_{\mathrm{b}}\right)$ $=\gamma_{\mathrm{m}}$ is reached before the internalization of the particle. The maximal increase in friction $\gamma_{\mathrm{m}} \approx \mathrm{pN} \mathrm{ms} \mu \mathrm{m}^{-1}$ corresponds to a relative increase of $70 \%$.

3.3.3. Local and global effects on particle uptake. In the sections above and in our mathematical model we have pointed out that the forces $F_{\text {def }}$ and energies $G_{\text {def }}$ required to insert a particle into a GUV are controlled by global and local deformation effects. Those can be better visualized by normalizing the curves $G_{\mathrm{def}}(h)$ and $F_{\mathrm{def}}(h)=-\frac{\mathrm{d}}{\mathrm{d} y} G_{\mathrm{def}}(h)$ in amplitude and in uptake length, here called relative piezo-movement $\beta \in[0,1]$, as displayed in Fig. 7. In this way, the slopes of all curves can be well compared. $\beta=1$ corresponds to the uptake length $h_{\mathrm{ut}}$, i.e. the maximal indentation length, where the particle is taken up. We investigated four different GUVs made from Egg-PC. Fig. 7a shows their normalized energy profiles, which are characterized by a shallow energy increase likely due to a global, elliptical deformation of the GUVs, followed by a steeper increase likely due to a local deformation caused by the indenting particle. The black arrow to the left indicates the increasing GUV radius $R_{\mathrm{GUV}}$ together with an earlier and smoother increase in deformation energy, likely due to a stronger influence of global deformation. By averaging over local and global effects, the energy profile can be approximated to be quadratic, $G_{\text {def }, n}(\beta) \approx C \beta^{2}$. Fig. $7 \mathrm{~b}$ displays the corresponding normalized force profiles $F_{\text {def }, n}(\beta)$, which, according to their slope, can be separated into ranges for global and local deformation. The latter comprises roughly $40 \%$ of the overall uptake length $(\beta=1)$.

The characteristics of the induced uptake process are influenced by the properties of the GUV-particle complex. In the experiments, the vesicle radius $R_{\mathrm{g}}$, the membrane tension $\sigma$ and, less obvious, the bending modulus $K$ can be varied. ${ }^{28}$ As described in Section 2.2, the membrane tension $\sigma$ has been reduced by the induced osmotic pressure difference in the sample. Due to the different GUV volumes, the resulting membrane tensions $\sigma$ will also be different in each case.
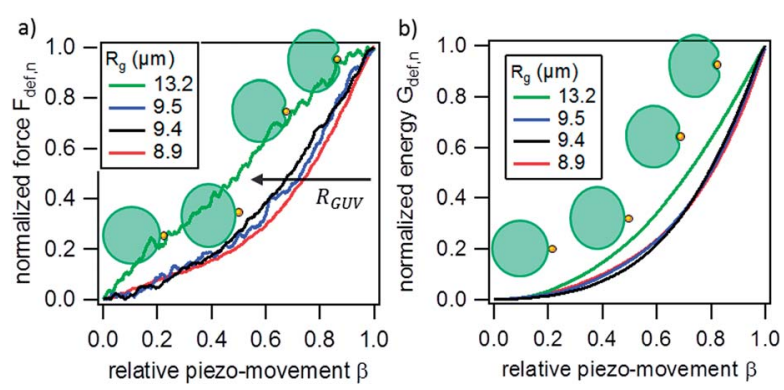

Fig. 7 Normalized uptake curves for different vesicle sizes. The radii increase from right to left. The piezo-displacement is normalized to the uptake length $s$ and thus called relative piezo-movement $\beta$. (a) The deformation is scaled to the respective uptake energy $G_{u t}$ and (b) the deformation force is related to $F_{\text {ut }}$.
3.3.4. Uptake energies and forces. Various purely physical parameters influence the uptake process as shown by our model, or vice versa, the uptake process can be characterized by mechanical parameters. Fig. 8a shows a comparison between measured uptake energies $G_{\mathrm{ut}}$, uptake forces $F_{\mathrm{ut}}$, membrane stiffnesses $\Delta \kappa_{\mathrm{m}}$ and membrane frictions $\Delta \gamma_{\mathrm{m}}$ for three of the four different Egg-PC vesicles. Whereas GUV no.1 and 2 have the same radius $R_{\mathrm{g}} \approx 9 \mu \mathrm{m}$, their bio-mechanical properties are quite different. The membrane of vesicle no. 2 has a 4 -fold higher radial stiffness $\left(\Delta \kappa_{\mathrm{m}} \approx 38 \mathrm{pN} \mu \mathrm{m}^{-1}\right)$ and 2-fold higher radial friction $\Delta \gamma_{\mathrm{m}}$ obtained from the fluctuation analysis of the bead pressed into the GUV. At the same time we measured a 2-fold higher uptake force $F_{\text {ut }}$ and a 2-fold higher uptake energy $G_{\mathrm{ut}}=\int F_{\mathrm{ut}}(h) \mathrm{d} h$. Here, $h$ designates the uptake length.

The dependency between the membrane tension $\sigma$ and the membrane stiffness $\Delta \kappa_{\mathrm{m}}$ was further manifested by the results of Fig. 8b, where the radial GUV membrane fluctuations were measured directly in the centre of the focused laser (without bead) similar to the approach presented in ref. 28. Here, the membrane tension $\sigma$ was estimated from the membrane
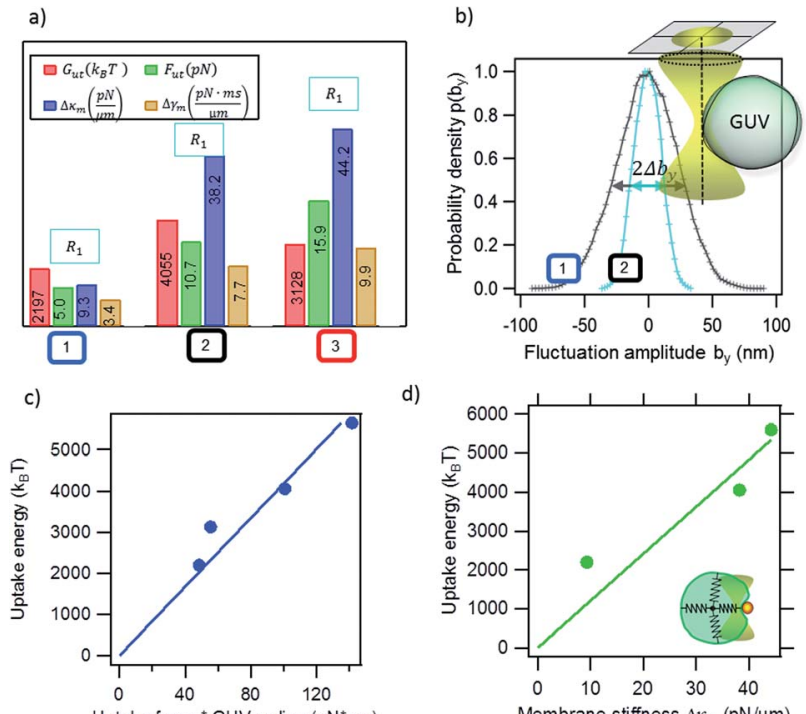

d)

e) Uptake force * GUV radius $\left(\mathrm{pN}^{*} \mu \mathrm{m}\right)$

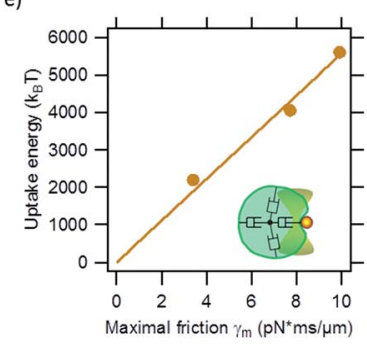

f)
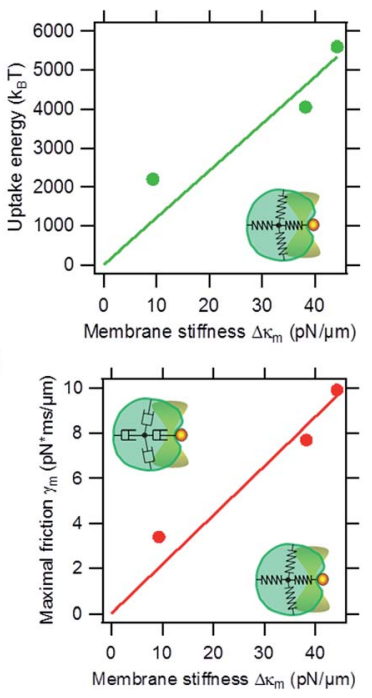

Fig. 8 Influence of mechanical parameters on the uptake process. (a) Comparison of uptake energy, uptake force, membrane stiffness and membrane friction for three different Egg-PC vesicles. (b) Histogram of the radial membrane fluctuations for vesicle no. 1 and 2 indicating different fluctuation widths $\Delta b_{y}$ and membrane tensions $\sigma$. (c) Uptake energies relative to the product of the uptake force and GUV radius. (d) Uptake energies relative to the membrane stiffness. (e) Uptake energies relative to the friction coefficient. (f) Maximal friction coefficient relative to the membrane stiffness. 
fluctuation width $\Delta b_{y}$. Vesicle no. 2 (black curve in Fig. $5 \mathrm{~d}$ ) has the same size as vesicle no. 1 (blue curve), but reveals a 2 -fold reduced fluctuation width $\Delta b_{y}$ and a 4-fold increased membrane stiffness $\Delta \kappa_{\mathrm{m}}$. As expected, a lower fluctuation width $\Delta b_{y}$ results in a higher effective membrane tension $\sigma$. Considering the blue and the black uptake curves in Fig. 5d, the GUV with the lower $\sigma$ results in a longer uptake length $h$ and a reduced uptake energy $E_{\mathrm{ut}}$.

These findings are generalized in Fig. 8c-f. Fig. 8c displays the linear relationship of the final uptake energy and the product of the uptake force and the vesicle radius. The uptake energy $G_{\mathrm{ut}}$ as a function of the uptake force times the uptake length, $F_{\mathrm{ut}} \times h_{\mathrm{ut}}$, results in a non-linear relationship due to the non-linear force profiles (not shown).

It can be seen in Fig. 8d and e that the uptake energy is approximately proportional to the membrane stiffness $\Delta \kappa_{\mathrm{m}}$ and to the maximal lateral friction coefficient $\gamma_{\mathrm{m}}$ briefly before internalization of the particle. In consequence, also the friction coefficient and the membrane stiffness have to be linear to each other as displayed in Fig. 8f.

\section{Discussion}

The goal of the present study has been the development and manifestation of a novel optical manipulation and measurement technique to study phagocytosis in a simple biomimetic system such as a GUV. It was our aim to obtain precise energy and force profiles together with supplementary physical parameters during phagocytosis, which we induced by optical tweezers and recorded by interferometric $3 \mathrm{D}$ particle tracking. It was a further aim to better understand the measured profiles by using an elastic deformation model considering global and local deformation effects due to indentation of a $1 \mu \mathrm{m}$ large bead.

\subsection{BFP particle tracking near the membrane}

The $1064 \mathrm{~nm}$ laser beam was focused by a NA $=n \sin \alpha=1.2$ water immersion objective lens resulting in a focusing angle of nearly $\alpha=64^{\circ}$. If the optical trapped $1 \mu \mathrm{m}$ latex particle is brought into contact with GUVs, which are 18-27 $\mu \mathrm{m}$ in diameter, the focused laser light will also hit the GUV. A first question to answer was whether the light scattered at the GUV could alter the optical trap and the particle tracking precision in the back focal plane (BFP) of the detection lens. The effect can be understood by the three beam interference leading to an additional intensity $\Delta I(x, y, d)$ in the focus and in the BFP $\Delta I\left(k_{x}, k_{y}, d\right)$, which depends on the distance between the focus and the GUV. By inspecting Fig. 1c, one can see that the most significant changes in the particle position signals occur in the radial $y$ direction, thus reducing the dimensions of the problem. It is demonstrated in Fig. 3 that these signals indeed represent the 3D particle position with a negligible small error of less than $10 \%$, since the bipolar position signal of the GUV membrane is significantly smaller in amplitude than the signal resulting from the latex bead. Furthermore, a change in the laser intensity did not result in a position change of the free-floating GUV, meaning that optical forces on the GUV are negligible.

\subsection{Mathematical deformation model}

Our model estimates the deformation energy $G_{\text {def }}$ of a spherical lipid bilayer sheet, which can be separated into energy components for bending and stretching. These can be further separated into a global deformation, where the spherical GUV is transformed into an oblate spheroid and into the local deformation, where the indentation of the spherical bead leads to a small spherical deformation across the contact area of the bead and a toroidal deformation around this area. In both cases the change in membrane curvature as well as the change in membrane area had to be calculated. By using appropriate coordinate systems and variables, the expressions for these four energy terms could be reduced to handy expressions. Our approach is similar to that of Deserno et al., ${ }^{33}$ although they present results in a more generalized way $\left(e . g\right.$. by $\left.\Lambda^{2}=K / \sigma\right)$. The final energy profile $G_{\mathrm{def}}(h)$ predicted by our model is a function of the local and global indentation height $h=h_{\mathrm{lo}}+h_{\mathrm{gl}}$. The model considers the GUV radius (here $R_{\mathrm{g}}=9 \mu \mathrm{m}$ and $\left.13 \mu \mathrm{m}\right)$, the bead radius $\left(R_{\mathrm{b}}=0.5\right.$ $\mu \mathrm{m})$, the membrane bending modulus for Egg-PC $\left(K_{0}=10 k_{\mathrm{B}} T\right)$, a

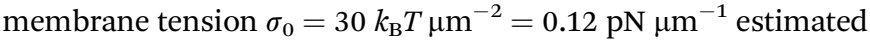
from the measured tether force $F_{\text {teth }}=8 \pi \sqrt{K \sigma}$, and the radius of the concave toroidal curvature from the local indentation $\left(R_{\mathrm{c}}=2.5 \mu \mathrm{m}\right)$, which however hardly affects the results.

The experimental parameters used correspond to a low tension membrane where $R_{\mathrm{g}} \approx \Lambda=0.57 \mu \mathrm{m}$ and with a reduced membrane tension $\tilde{\sigma}_{0}=R_{\mathrm{b}}{ }^{2} \sigma_{0} / K_{0}=\left(R_{\mathrm{b}} / \Lambda\right)^{2}=0.77$, which is close to those expected for cellular membranes. ${ }^{19}$

In the simulations both $K$ and $\sigma$ were varied by a factor of 4 to investigate the influence on the energy profile, which changes significantly more by reducing $\sigma$. In other words, the reduced membrane tension results in a longer uptake length which was also shown in the experiments, where vesicle no. 1 (blue curve) has a four times slower membrane stiffness $\Delta \kappa_{\mathrm{m}}$ relative to vesicle no. 2 (black curve). The strength of the model is that for reasonably estimated parameters $K$ and $\sigma$, both the profile slope and the absolute deformation energies coincide well with the experiment. From Fig. $5 \mathrm{~d}$ we could estimate the uptake length to be $h_{\mathrm{ut}}=R_{\mathrm{g}} / 3+2 R_{\mathrm{b}}$, where the local indentation height $2 R_{\mathrm{b}}=1$ $\mu \mathrm{m}$ corresponds to complete wrapping of the particle. The global critical indentation height $R_{\mathrm{g}} / 3$ varies with the vesicle radius. The calculated energies $G_{\mathrm{ut}}=G_{\mathrm{def}}\left(h_{\mathrm{ut}}\right)$ at a typical uptake length of $h_{\mathrm{ut}}=R_{\mathrm{g}} / 3+1 \mu \mathrm{m}$ are all in the range of some $1000 k_{\mathrm{B}} T$.

The simulated energy profile for a local deformation (orange curve in Fig. 5e), although plotted over the whole range of $5 \mu \mathrm{m}$, does not reach values larger than the particle diameter $=1 \mu \mathrm{m}$. According to our theoretical model, the required energy for an estimated uptake increases nonlinearly with the vesicle radius $R_{\mathrm{g}}$ as shown in Fig. 5f. The increase is smaller for larger ratios $\Lambda^{2}=K / \sigma$.

The superposition of the local and global deformation energies confirm our mechanistic model and understanding that particle engulfment and uptake first lead to a global deformation followed by a local GUV deformation. The scale of the indentation lengths $h_{\mathrm{lo}}$ and $h_{\mathrm{gl}}$ are based on the harmonic approximation of the energy profiles, and use the concepts of serial connected spring constants, which add inversely. This serial connection of potential landscapes needs to be improved 
in the future if the energy profiles $G_{\mathrm{def}}(h)$ become more complicated. Useful next steps would be to include the energies for tether formation and membrane rupturing in our model. In summary, our model represents a valuable approach to validate the observation, which can be extended and improved as proposed by others. ${ }^{19-21,34}$

\subsection{Induced phagocytosis}

The particle fluctuations in the vicinity of the membrane are recorded at the very high sampling rate of $1 \mathrm{MHz}$, enabling the investigation of the contact process between the particle and the GUV - before and after close contact with the membrane. As soon as the particle come into contact with the membrane, the particle's position fluctuations change both in amplitude and time, which can be analysed by the fluctuation width or the stiffness $\kappa$. In the case of sudden binding to the membrane, the fluctuation width changes instantaneously. ${ }^{27,40}$ However, Fig. 4 reveals a smooth increase in $\kappa$ in the radial direction, indicating that the particle is slowly pressed against the membrane leading to a slow decrease of the fluctuation width.

4.3.1. No adhesion energy. Since the particle did not bind to the membrane, no adhesion energy could be exploited to reduce the required uptake energy. A possible reason for this is that the particle has already been coated with lipids thus blocking a possible van der Waals interaction. This increase of the radial and tangential friction coefficient has been measured for the first time and resembles the behaviour of a particle in front of a stiff wall. Furthermore the increase in radial friction is consistent with the results obtained with other techniques such as optical dynamometry. ${ }^{\mathbf{4 2 , 4 3}}$ In future experiments the influence of adhesion energies should be estimated by approaching uncoated particles to the membrane.

4.3.2. Energetic steps during phagocytic uptake. We have demonstrated that the induced uptake of the particle into a GUV is initiated by a global deformation followed by a local deformation, which results in an initial smooth increase of the deformation energy followed by a steeper energy increase during local indentation. On a comparable indentation length, the first step requires less energy, but might give the (biomimetic) cell time to reach thermal equilibrium after reorganization of membrane (or cytoskeleton) structures. The second step of indentation, the stretching of the membrane, seems to require considerably more energy, until the particle is completely taken up. It is likely that this amount of energy is controlled by the lipid reorganization during the first step of membrane bending. A last energetic step can be observed during a sudden drop off in force and energy after a typical indentation length of about $20 \%$ of the GUV diameter. The process is visible by video microscopy and QPD tracking in parallel. Similar global and local deformations of the floppy GUV had been imaged and presented in previous studies. ${ }^{\mathbf{4 4}}$ However, it needs to be noted that the elastic GUV is squeezed between a large $20 \mu \mathrm{m}$ glass bead and the $1 \mu \mathrm{m}$ optical trapped bead leading to a global deformation of the GUV. In real cellular phagocytosis the effects of the global deformation are expected to be less relevant.
4.3.3. Membrane fluctuations as an indicator for phagocytic efficiency. For the first time it has become possible to measure energies and forces for a particle uptake into a GUV. These are in the range of $2000-5500 k_{\mathrm{B}} T$ and 3-16 pN, respectively. Our values are significantly higher than those predicted by Deserno et al., ${ }^{19}$ where uptake energies of $22 k_{\mathrm{B}} T$ and $66 k_{\mathrm{B}} T$ are provided for GUVs with a comparable reduced membrane tension $\tilde{\sigma}_{0}=0.22$ and $\tilde{\sigma}_{0}=1$, respectively. These values vary with the GUV size and the maximal membrane stiffness $\Delta \kappa_{\mathrm{m}}$, which have been evaluated from the fluctuations of the membrane alone or with the bead attached (Fig. 8a and b). As shown by Betz ${ }^{28}$ and confirmed by us in Fig. $8 \mathrm{~b}$, the membrane tension $\sigma$ increases with the inverse membrane fluctuation width $1 / \Delta b_{y}$. In a proof-of-principle experiment we have demonstrated the increase of the membrane tension through $1 / \Delta b_{y}$ with the membrane stiffness $\Delta \kappa_{\mathrm{m}}$. However, further experiments and theoretical considerations have to be performed to understand the relationship between both quantities.

This increase of the uptake energy with the membrane tension has been theoretically predicted by several authors. ${ }^{19,21,34}$ This prediction could be validated by the results shown in Fig. 8d, where uptake energies increase linearly with the membrane stiffness $\kappa_{i}=k_{\mathrm{B}} T /\left\langle b_{i}{ }^{2}\right\rangle$, which is the inverse squared fluctuation width $\left\langle b_{i}{ }^{2}\right\rangle$ of the fully wrapped bead briefly before penetration. The physical and quantitative dependency between this membrane fluctuation parameter and the membrane tension $\sigma$ still need to be evaluated. Interestingly, the uptake energy can also be estimated by analyzing the wrapped particle fluctuations on a very short time scale, resulting in the linear dependency to the radial friction coefficient $\gamma_{\mathrm{m}}$ as shown in Fig. 8e. Motions of the wrapped particle are strongly damped before the final uptake. Although the linear slope shown in Fig. 8f, can be concluded from the other two figures, this result is also far from being obvious and might be helpful for future uptake experiments.

It should be mentioned that biological cell membranes are characterized by unique permeability and adaptive mechanical properties, which means that under physiological conditions biomembranes are on the melting point, which means that they are on the switch between the gel and liquid-crystalline phase. ${ }^{4,46}$ The Egg-PC membranes we used have a relatively low melting point such that they are in the melted phase, such that no additional energy for melting is required. However, it remains an open question how the phase of the membrane influences their viscoelastic properties and the final uptake forces and energies.

Based on our experimental procedure and the present analysis, it should be possible to compare uptake energy profiles from different GUVs with more complex membrane compositions. Even systems with protein induced membrane deformations ${ }^{\mathbf{1 2}}$ could be compared allowing one to investigate phagocytosis on a macromolecular level.

\section{Conclusion}

We succeeded in inducing the phagocytic uptake of a particle into a giant unilamellar vesicle (GUV) by means of optical tweezers. 
With increasing indentation length of the particle into the membrane, we measured membrane deformation energies that can be separated into two subsequent steps. We found uptake energies of 2000-5500 $k_{\mathrm{B}} T$ for GUVs with different sizes and membrane tensions. Adhesion energies between the particle and the GUV that could lower the uptake energies were blocked, facilitating the analysis of global and local deformation energies of the GUV. In principle, the above-mentioned uptake energies can be brought up by conformational transitions of membrane-bound proteins. These uptake energies can be lowered by avoiding a global deformation of the GUV, as it is likely the case for biological cells. But, and this keeps this field of research very exciting, the uptake energies can be purely delivered by accessible adhesion energies between the particle and the host artificial cell. Our experimental procedure and theoretical model presented in this study should be applicable to other experiments as well with step-wise increased complexity, thus enabling a bottom-up strategy to understand the physics of phagocytosis.

\section{Acknowledgements}

The authors thank Dr Timo Betz for helpful comments and discussions as well as Prof. Dieter Braun for general support. B. Tränkle was supported by the Deutsche Forschungsgemeinschaft, grant number RO 3615/1. W. Römer was supported by the Excellence Initiative of the German Research Foundation (EXC 294), by a grant from the Ministry of Science, Research and the Arts of Baden-Württemberg (Az: 33-7532.20) and by a starting grant of the European Research Council (Programme "Ideas" no. ERC-2011-StG 282105).

\section{References}

1 P. Cossart and P. J. Sansonetti, Science, 2004, 304, 242-248.

2 T. Eierhoff, B. Stechmann, W. Römer, Pathogen and Toxin Entry - How Pathogens and Toxins Induce and Harness Endocytotic Mechanisms, in Molecular Regulation of Endocytosis, ed. B. Ceresa, InTech, 2012, pp. 249-256.

3 B. J. Reynwar, G. Illya, V. A. Harmandaris, M. M. Müller, K. Kremer and M. Deserno, Nature, 2007, 447, 461-464.

4 E. A. Evans and D. A. Calderwood, Science, 2007, 316, 11481153.

5 H. Kress, J. G. Park, C. O. Mejean, J. D. Forster, J. Park, S. S. Walse, Y. Zhang, D. Q. Wu, O. D. Weiner, T. M. Fahmy and E. R. Dufresne, Nat. Methods, 2009, 6, 905-909.

6 J. A. Champion and S. Mitragotri, Proc. Natl. Acad. Sci. U. S. A., 2006, 103, 4930-4934.

7 S. E. A. Gratton, P. A. Ropp, P. D. Pohlhaus, J. C. Luft, V. J. Madden, M. E. Napier and J. M. DeSimone, Proc. Natl. Acad. Sci. U. S. A., 2008, 105, 11613-11618.

8 S. Tollis, A. E. Dart, G. Tzircotis and R. G. Endres, BMC Syst. Biol., 2010, 4, DOI: 10.1186/1752-0509-4-149.

9 H. Kress, E. H. Stelzer, D. Holzer, F. Buss, G. Griffiths and A. Rohrbach, Proc. Natl. Acad. Sci. U. S. A., 2007, 104, 11633-11638.
10 R. Dimova, S. Aranda, N. Bezlyepkina, V. Nikolov, K. A. Riske and R. Lipowsky, J. Phys.: Condens. Matter, 2006, 18, S1151.

11 H.-G. Döbereiner, O. Selchow and R. Lipowsky, Eur. Biophys. J., 1999, 28, 174-178.

12 P. Sens, L. Johannes and P. Bassereau, Curr. Opin. Cell Biol., 2008, 20, 476-482.

13 D. L. Richmond, E. M. Schmid, S. Martens, J. C. Stachowiak, N. Liska and D. A. Fletcher, Proc. Natl. Acad. Sci. U. S. A., 2011, 108, 9431-9436.

14 W. Römer, L. Berland, V. Chambon, K. Gaus, B. Windschiegl, D. Tenza, M. R. E. Aly, V. Fraisier, J. C. Florent, D. Perrais, C. Lamaze, G. Raposo, C. Steinem, P. Sens, P. Bassereau and L. Johannes, Nature, 2007, 450, 670-U673.

15 W. Römer, L.-L. Pontani, B. Sorre, C. Rentero, L. Berland, V. Chambon, C. Lamaze, P. Bassereau, C. Sykes and K. Gaus, Cell, 2010, 140, 540-553.

16 K. Jaskiewicz, A. Larsen, D. Schaeffel, K. Koynov, I. Lieberwirth, G. Fytas, K. Landfester and A. Kroeger, ACS Nano, 2012, 6, 7254-7262.

17 C. Dietrich, M. Angelova and B. Pouligny, J. Phys. II, 1997, 7, 1651-1682.

18 U. Seifert, Adv. Phys., 1997, 46, 13-137.

19 M. Deserno, Phys. Rev. E: Stat. Phys., Plasmas, Fluids, Relat. Interdiscip. Top., 2004, 69, 031903.

20 C. C. Fleck and R. R. Netz, Europhys. Lett., 2004, 314.

21 S. Dasgupta, T. Auth and G. Gompper, Soft Matter, 2013, 9, 5473-5482.

22 A. H. Bahrami, Soft Matter, 2013, 9, 8642-8646.

23 A. Rohrbach, Opt. Express, 2005, 13, 9696-9701.

24 A. Jannasch, A. F. Demirors, P. D. J. van Oostrum, A. van Blaaderen and E. Schaffer, Nat. Photonics, 2012, 6, 469-473.

25 A. Pralle, M. Prummer, E.-L. Florin, E. H. K. Stelzer and J. K. H. Hörber, Microsc. Res. Tech., 1999, 44, 378-386.

26 L. Friedrich and A. Rohrbach, Opt. Lett., 2012, 37, 21092111.

27 H. Kress, E. H. Stelzer, G. Griffiths and A. Rohrbach, Phys. Rev. E: Stat. Phys., Plasmas, Fluids, Relat. Interdiscip. Top., 2005, 71, 061927.

28 T. Betz and C. Sykes, Soft Matter, 2012, 8, 5317-5326.

29 M. I. Angelova and D. S. Dimitrov, Faraday Discuss. Chem. Soc., 1986, 81, 303-311.

30 L. Mathivet, S. Cribier and P. F. Devaux, Biophys. J., 1996, 70, 1112-1121.

31 A. Rohrbach, C. Tischer, D. Neumayer, E. L. Florin and E. H. K. Stelzer, Rev. Sci. Instrum., 2004, 75, 2197-2210.

32 W. Helfrich, Z. Naturforsch., C: Biochem., Biophys., Biol., Virol., 1973, 28, 693-703.

33 M. Deserno and W. M. Gelbart, J. Phys. Chem. B, 2002, 106, 5543-5552.

34 M. Deserno and T. Bickel, EPL, 2003, 62, 767.

35 M. Speidel, L. Friedrich and A. Rohrbach, Opt. Express, 2009, 17, 1003-1015.

36 M. Koch and A. Rohrbach, Nat. Photonics, 2012, 6, 680-686. 37 J. Lemiere, K. Guevorkian, C. Campillo, C. Sykes and T. Betz, Soft Matter, 2013, 9, 3181-3187.

38 A. Rohrbach, Simulation software LightWave 1.1, 2013. 
39 P. C. Seitz, E. H. Stelzer and A. Rohrbach, Applied Optics, 2006, 45, 7309-7315.

40 F. Jünger, F. Kohler, A. Meinel, T. Meyer, R. Nitschke, B. Erhard and A. Rohrbach, Biophys. J., in preparation.

41 I. Derényi, F. Jülicher and J. Prost, Phys. Rev. Lett., 2002, 88, 238101.

42 R. Dimova, C. Dietrich, A. Hadjiisky, K. Danov and B. Pouligny, Eur. Phys. J. B, 1999, 12, 589-598.
43 R. Dimova, B. Pouligny and C. Dietrich, Biophys. J., 2000, 79, 340-356.

44 I. Koltover, J. O. Rädler and C. R. Safinya, Phys. Rev. Lett., 1999, 82, 1991.

45 T. Baumgart, S. T. Hess and W. W. Webb, Nature, 2003, 425, 821-824.

46 O. G. Mouritsen, Eur. J. Lipid Sci. Technol., 2011, 113, 11741187. 